\title{
Glaucoma in Fuchs' Heterochromic Uveitis: Aetiology, Management and Outcome
}

\author{
NICHOLAS P. JONES
}

\begin{abstract}
Summary
A study of glaucoma in a cohort of 103 patients with Fuchs' Heterochromic Uveitis (FHU) is reported. Twenty-seven patients $(\mathbf{2 6 . 2 \%})$ had glaucoma. Half of these had glaucoma on presentation. The risk of development of glaucoma after presentation with FHU is $0.5 \%$ per year, falling substantially after 15 years follow-up. Causes of glaucoma include inflammation with peripheral anterior synechiae, rubeosis, lensinduced angle closure and recurrent spontaneous hyphaema, but most patients had chronic open angle glaucoma. Cataract surgery may precipitate glaucoma. Most patients were managed medically. The failure rate of glaucoma drainage surger:' was $55.5 \%$, and 5-Fluorouracil is to be recommended as an adjunct to surgery.
\end{abstract}

In 1906 Ernst Fuchs comprehensively reported $^{1}$ on a series of 38 patients with 'complicated heterochromia'. In this original series, three of these patients $(7.9 \%)$ were

noted to have glaucoma. It is now recognised that of all the features of Fuchs' heterochromic uveitis (FHU), it is the glaucoma that is most troublesome. Opinions on its prevalence have varied markedly, though it is now seen that the figure in Fuchs' original series was low. More recent estimates vary from $9 \%^{2}$ to $59 \%$. $^{3}$

Reports on the aetiology of glaucoma in FHU have variously included lens-induced acute glaucoma,${ }^{4}$ trabecular sclerosis, ${ }^{5}$ phakolysis, ${ }^{6}$ peripheral anterior synechia formation, ${ }^{7}$ neovascularisation, ${ }^{3,8-11}$ trabeculitis ${ }^{9}$ and steroid induction. ${ }^{6}$ The management of the glaucoma has been considered difficult, with drainage surgery being frequently necessary. ${ }^{3,6}$ The response to that surgery has been reported as being reliable ${ }^{12}$ or unreliable. ${ }^{6}$ The relationship of the glaucoma to cataract surgery, ${ }^{11,13-15}$ or to other intraocular procedures ${ }^{16}$ has been a matter for concern.
The aetiology and preferred management of glaucoma in FHU is by no means generally agreed. The author has studied a large cohort of 103 patients with FHU. ${ }^{17}$ of whom a pro-

portion have glaucoma. The results of observations on the aetiology, presentation and course of the glaucoma, and its response to medical and surgical management, are presented.

\section{Patients and methods}

From 1987, all new and old patients seen at Manchester Royal Eye Hospital, with a diagnosis of FHU, were seen by the author. Some patients were found to have, or have had, glaucoma. Both acute and chronic glaucoma were seen. Acute glaucoma is, for the purposes of this survey, defined as a precipitate rise in intraocular pressure requiring temporary antiglaucoma medication; chronic glaucoma is defined as a sustained intraocular pressure rise with evidence of optic disc and visual field damage, or in the absence of this evidence, a sustained very high intraocular 
pressure rise $(>40 \mathrm{mmHg})$. All patients were examined by the author, most of these being examined on several occasions. Features of the disease with relevance to the glaucoma are reported.

\section{Results}

One hundred and three patients with FHU were studied. The diagnosis was made by a combination of physical signs, including the pattern of insidious intraocular inflammation with characteristic keratic precipitates, iris depigmentation and atrophy, and complicated cataract. Of these 103 patients, 27 were found to have glaucoma according to the above criteria $(26.2 \%)$. All but one of these glaucomatous patients had unilateral FHU. In addition, four patients had been noted to have an unsustained rise in intraocular pressure on one or more occasions, but had no evidence of optic disc cupping or visual field loss and had not required anti-glaucoma medication. These patients did not fulfil the criteria outlined above and were not included in this report.

Of the 27 patients with glaucoma, 20 were female, and seven male. This discrepancy is significant $(\mathrm{p}=0.02)$. The mean age at presentation was 43.2 years (range $20-62$ years), compared to a mean of 36.4 years (range 7-71 years) for the whole cohort of 103 patients. This difference is also significant at the level $\mathrm{p}=0.02$. The mean follow-up time since presentation was 10.2 years, with a range of 3-24 years.

The mean age at diagnosis of glaucoma was 46.1 years (range 29-63 years). In 14 of the 27 patients $(51.8 \%)$ the glaucoma was diagnosed on presentation to an ophthalmologist, the remaining 13 patients developing glaucoma later. The risk, for patients with FHU, of developing glaucoma after presentation, was $0.5 \%$ per year for the first fifteen years. Only one patient developed glaucoma more than 15 years after presentation (one of 20 patients who have been followed for this period of time).

The causes of the glaucoma are shown in Table I. Initially, 21 patients $(77.7 \%$ of those with glaucoma) appeared to have chronic open angle glaucoma (COAG). However, the gonioscopic appearance changed with time in a number of patients, especially after intraocular surgery or episodes of increased inflammation, and the eventual main cause of glaucoma is also shown in Table I. To date, six patients have developed iris surface and angle rubeosis. Peripheral anterior synechia (PAS) formation was common in this series, though the significance of this in relation to glaucoma was unclear expect in those four cases in which it became gross.

One patient presented with acute lensinduced angle closure glaucoma due to an intumescent cataract. Another patient developed recurrent acute glaucoma due to repeated spontaneous hyphaemas. Many of the 103 patients received topical steroid medication at some stage in their disease, either before the diagnosis of FHU was made, or later. In many cases (owing to the loss of previous hospital records) the timing and quantity of steroid treatment could not be ascertained. No correlation could be fọund between previous steroid treatment and the development of glaucoma, except after cataract surgery, where the cause may be multifactorial.

In this series of 27 patients with glaucoma, 15 have undergone cataract surgery. In eight cases the cataract operation was followed by the development of glaucoma, and in four cases with pre-existing glaucoma, the glaucoma became more difficult to control after cataract surgery. In two cases, the cataract surgery appeared to have no effect on the glaucoma, and in the remaining case topical glaucoma medication was stopped and intraocular pressure remained normal. Of the 15 eyes undergoing cataract surgery, five eyes underwent intracapsular cataract extraction (ICCE). Three of these eyes have been enucleated (two had iris-supported IOLs). One other eye was enucleated, and this eye was phakic. No eye undergoing extracapsular surgery has been lost, but follow-up on these patients is shorter.

Six patients have required trabeculectomy to date. Four other patients developed painful blind eyes with rubeosis, PAS and corneal decompensation. These four eyes have been enucleated. The remaining 17 patients are stable on topical anti-glaucoma medication.

Of those six patients who underwent trabe- 
Table I. The causes of glaucoma

\begin{tabular}{lcc}
\hline & \multicolumn{2}{c}{ Number of patients } \\
\cline { 2 - 3 } Cause & At first diagnosis & Now \\
\hline Chronic Open-Angle & 21 & 16 \\
Peripheral Anterior Synechiae & 1 & 4 \\
Rubeosis & 3 & 6 \\
Lens-induced angle closure & 1 & 0 \\
Recurrent spontaneous hyphaema & 1 & 1 \\
\hline Total & 27 & 27 \\
\hline
\end{tabular}

culectomy, three are controlled satisfactorily after a single procedure (mean follow-up since surgery 27 months). The remaining three have undergone a total of nine glaucoma procedures; One patient, after two trabeculectomies failed due to bleb inadequacy, required a glaucoma drainage tube and cyclocryotherapy subsequent to this. The second patient has a satisfactorily-functioning trabeculectomy after the first failed due to bleb fibrosis, and the third, after failed trabeculectomy followed by failed combined surgery (trabeculectomy with cataract surgery), has a functioning third trabeculectomy (performed using 5-Fluorouracil). Again in this patient, bleb failure was the reason for further surgery.

The present visual acuities for the 27 affected eyes are shown in Table II. Some of these patients have cataract and/or vitreous opacification, therefore are compared with the present visual acuities for the other 77 FHU patients, who do not have glaucoma but who may also have cataract or vitreous opacification. For those patients with glaucoma, only $33.3 \%$ have a visual acuity of $6 / 12$ or better, compared to a figure of $73.9 \%$ for those without glaucoma.

\section{Discussion}

\section{A. The Aetiology of Glaucoma in FHU}

It is clear that a rise in intraocular pressure in FHU may be caused by one of several factors. It is also clear that more than one fact or may be present simultaneously, and that the aetiology may change with time. The glaucomas in this series fall into distinct groups:

\section{Glaucoma associated with uveitis}

There may be occasional exacerbations of anterior uveitis in FHU, and increased inflammation is certainly a factor after some cataract operations. The development of PAS may follow such exacerbations, may be combined with rubeosis, or may follow after many years of an indolent anterior segment inflammation. In this survey, there was evidence of significant PAS in only four patients with glaucoma, and in only one of these did it appear to be the initial cause of the glaucoma.

\section{Glaucoma associated with neovascularisation}

The existence of neovascularisation of the iris stroma and anterior chamber angle in some patients with FHU is beyond dispute. Saari et

Table II. Present visual acuities

\begin{tabular}{|c|c|c|c|}
\hline \multirow[b]{2}{*}{ Visual acuity } & \multicolumn{2}{|c|}{ FHU with glaucoma } & \multirow{2}{*}{$\frac{\text { FHU without glaucoma }}{(\%)}$} \\
\hline & Number & $(\%)$ & \\
\hline$\geqslant 6 / 6$ & 3 & 11.1 & 29.3 \\
\hline $6 / 9-6 / 12$ & 6 & 22.2 & 44.6 \\
\hline $6 / 18-6 / 24$ & 6 & 22.2 & 16.9 \\
\hline $6 / 36-6 / 60$ & 5 & 18.6 & 4.6 \\
\hline$<6 / 60$ & 7 & 25.9 & 4.6 \\
\hline (NPL) & (4) & (14.8) & $(0.0)$ \\
\hline Total & 27 & 100.0 & 100.0 \\
\hline
\end{tabular}


$a l^{18}$ have demonstrated neovascularisation and leakage from the iris stroma in patients with FHU, using anterior segment fluorescein angiography. The prevalence of such changes is however a matter for discussion. Some authors have found a high proportion of irides with neovascularisation and vascular abnormalities in the anterior chamber angle..$^{3,7,19,20}$ Others $^{2}$ have found only small proportions of such patients and the supposed frequency of such changes has been questioned. ${ }^{21}$ Henkind ${ }^{22}$ has discussed the normal variation in anterior chamber angle vasculature and has discussed the implications for FHU, concluding that many of such observations were in fact normal variants.

Close examination of the iris in FHU reveals extensive loss of anterior border layer and stromal pigment, and loss of stromal volume. Both normal and abnormal vasculature therefore assume a greater prominence, and neovascularisation should be easily visible with slit-lamp biomicroscopy. Rubeosis was seen in only six patients in this series, either after cataract surgery (four patients) or arising spontaneously (two patients). The resultant glaucoma was responsible for four enucleations. In none of these patients was there evidence of another cause of rubeosis, such as posterior segment vascular occlusion.

\section{Glaucoma associated with cataract formation}

Cataract formation is virtually universal in $\mathrm{FHU}^{3,23}$ and therefore any lens-induced glaucoma is possible. Progression to hypermaturity may be rapid. One patient in this series had acute angle closure glaucoma induced by an intumescent lens. Although phakolysis was not seen in this series, it has been previously reported $^{6}$ and must be noted as a possible cause of glaucoma. Lens subluxation or dislocation may occur in any chronic uveitis. Phakodonesis has been noted in two patients in this cohort of 103 , and in one patient undergoing cataract surgery the lens spontaneously dislocated. The theoretical possibility of acute glaucoma induced by lens dislocation must therefore be added to this list.

\section{Glaucoma after cataract surgery}

The problem of glaucoma following cataract surgery in FHU has been addressed on several occasions. Ward and Hart ${ }^{24}$ reported in 1967 on nine patients with FHU who had suffered complications after cataract surgery. Six of these had glaucoma. Norn ${ }^{13}$ found that 11 of 23 eyes $(48 \%)$ undergoing ICCE had developed glaucoma and four of these eyes had been enucleated. Smith and O'Connor ${ }^{14}$ reported a series of 29 eyes undergoing ICCE or ECCE and found that four eyes (13.8\%) developed glaucoma. In one case this was transient. Liesegang, ${ }^{3}$ discussing the results of 17 cataract operations of unspecified type, found that four eyes $(23.5 \%)$ had developed glaucoma de novo after surgery, of which one was enucleated. Mills and Rosen reported in $1982^{25}$ on a group of eight eyes undergoing ICCE with IOL implantation. Two eyes developed glaucoma.

Three surveys of ECCE \pm IOL have been published. Gee and Tabbara ${ }^{26}$ reported in 1989 on 15 eyes (10 ECCE + IOL, 5 ECCE) and none of these eyes developed increased intraocular pressure during the follow-up period. This is in contrast to the findings of Jones $^{15}$ who, reporting on 30 eyes (20 ECCE + IOL, $10 \mathrm{ECCE})$, found that 10 (33.3\%) developed glaucoma (eight with IOL, two without). Five of these glaucomas were transient but five persisted. Jakeman et al, ${ }^{27}$ discussed the results of $20 \mathrm{ECCE}+\mathrm{IOL}$ operations. Four patients $(20 \%)$ developed glaucoma, of whom three required drainage surgery.

Hooper et al, in their review of cataract extraction in patients with uveitis ${ }^{28}$ comment that, as glaucoma is common in FHU anyway, the reported cases of glaucoma following cataract surgery may simply be a reflection of the natural history of the disease, implying that the surgery itself is irrelevant. The experience of this author does not support that hypothesis. Intraocular pressure can rise precipitately after cataract surgery, is clearly related to that surgery, and may require long-term treatment. It is probable that eyes which are predisposed to glaucoma, and which may have already been observed to have intermittent rises in intraocular pressure preoperatively, can be tipped over the edge into glaucoma by cataract surgery, which even in uncomplicated cases is a significant ocular 
insult, and which in those not infrequent cases complicated by a vigorous uveitis, can occlude the drainage angle by anterior synechiae. Glaucoma, and the special implications of glaucoma in the context of FHU, must be accepted as a common complication of cataract surgery in FHU and the possibility should be specifically discussed with the patient preoperatively, for informed consent.

\section{Chronic open angle glaucoma (COAG)}

The majority (18) of these patients developed glaucoma without any abnormal gonioscopic finding or other apparent cause of glaucoma, though some went on to develop PAS or rubeosis later. No underlying cause was found and no patient had a family history of glaucoma.

The administration of topical steroid treatment, sometimes over several years, was not uncommon in this cohort of 103 patients, yet no evidence of steroid induction of glaucoma was found. Severe, and sometimes sustained, rises of intraocular pressure were seen after cataract surgery but often arose within two weeks of the surgery, ${ }^{15}$ making steroid induction seem an unlikely cause of the glaucoma. This study does not elucidate the cause of the COAG in patients with FHU.

Only one patient had bilateral glaucoma (but unilateral FHU). Franceschetti ${ }^{29}$ felt that glaucoma was more common in those with bilateral FHU. That is not supported by this survey. Of the 103 patients with FHU, eight have bilateral disease. Only one of these has glaucoma, and that is unilateral, following cataract surgery.

\section{B. Management and Outcome}

Most patients $(17,63.0 \%$ ) have so far been managed medically and there is no indication from this series that medical management is any less likely to succeed in FHU than in other forms of glaucoma, despite previous reports to the contrary. ${ }^{3,6}$ Those patients coming to drainage surgery do appear however to be at increased risk of bleb failure (trabeculectomy failure rate in this series is $55.5 \%$ ). It would seem prudent to consider the use of 5-Fluorouracil in these patients, even for a first drainage procedure.

In comparison with FHU patients without glaucoma, the visual acuity in these patients is significantly affected. FHU is not necessarily a benign disease, and of these patients with glaucoma, four (14.8\%) have had (updated), enucleation for a painful blind eye.

Despite the high percentage of patients with FHU who have glaucoma $(25.5 \%)$, it is notable, and has not previously been reported, that more than half of these patients have glaucoma on presentation. The low subsequent conversion rate to glaucoma $(0.5 \%$ per year) is therefore surprising. Though annual or biennial tonometry by an optometrist should be recommended, routine follow-up of FHU patients without other problems is probably unnecessary. Most will return in the future with cataract in any case.

\section{Conclusions}

Glaucoma is a common complication of FHU, has several possible causes, and may be difficult to treat. It may be precipitated by cataract surgery and patients should be aware of this. Trabeculectomy is prone to failure and 5-Fluorouracil treatment may be necessary.

I am grateful to all doctors at Manchester Royal Eye Hospital for referring these patients.

Key Words: Fuchs' Heterochromic Uveitis Glaucoma

\section{References}

${ }^{1}$ Fuchs E: Ueber Komplicationen der Heterochromie. Z Augenheilk 1906, 15: 191-212.

2 Jain IS, Gupta A, Gangwar DN, Dhir SP: Fuchs' heterochromic cyclitis: some observations on clinical picture and on cataract surgery. Ann Ophthalmol 1983, 15: 640-2.

${ }^{3}$ Liesegang TJ: Clinical features and prognosis in Fuchs' uveitis syndrome. Arch Ophthalmol 1982, 100: $1622-6$.

${ }^{+}$Hart T: The IOP in Fuch's heterochromic cyclitis. Trans Ophthalmol Soc UK 1971, 91: 771-5.

${ }^{5}$ Huber A: Das Glaukom bei komplizierten Heterochromie Fuchs. Ophthalmologica 1961, 142: 66-115.

${ }^{6}$ Regenbogen LS and Naveh-Floman N: Glaucoma in Fuchs' heterochromic cyclitis associated with con:genital Horner's syndrome. $\mathrm{Br} J$ Ophthalmol 1987, 71: 844-9.

${ }^{7}$ O'Connor GR: Heterochromic iridocyclitis. Trans Ophthalmol Soc UK 1985, 104: 219-31.

${ }^{8}$ Berger BB, Tessler HH, Kottow MH. Anterior seg. ment ischaemia in Fuchs' heterochromic cyclitis. Arch Ophthalmol 1980, 98: 499-501.

${ }^{9}$ Perry HD, Yanoff M, Scheie HG: Rubeosis in Fuchs heterochromic iridocyclitis. Arch Ophthalmol 1975, 93: 337-9. 
${ }^{10}$ Lerman $S$ and Levy C: Heterochromic iritis and secondary neovascular glaucoma. Am J Ophthalmol 1964, 57: 479-81.

${ }^{11}$ Hart CT and Ward DM: Intra-ocular pressure in Fuchs's heterochromic uveitis. Br J Ophthalmol 1967, 51: 739-43.

${ }^{12}$ Grant WM: Heterochromic cyclitis (Fuchs). In: Chandler PA, Morton Grant W (Eds.) Glaucoma. Lea and Febinger, Philadelphia 1979.

${ }^{13}$ Norn MS: Cataract extraction in Fuchs' heterochromic: follow-up of 19 cases. Acta Ophthalmol 1968, 46: 685-99.

${ }^{14}$ Smith RE and O'Connor R. Cataract extraction in Fuchs' syndrome. Arch Ophthalmol 1974, 91: $39-41$.

${ }^{15}$ Jones NP: Extracapsular cataract surgery with and without intraocular lens implantation in Fuchs' heterochromic uveitis. Eye 1990, 4: 145-50.

${ }^{16}$ Roussel TJ and Coster DJ: Fuch's heterochromic cyclitis and posterior capsulotomy. Br J Ophthalmol 1985, 69: 449-51.

${ }^{17}$ Jones NP: Fuchs' Heterochromic Uveitis: A reappraisal of the clinical spectrum. Eye 1991: 5: 649-61.

${ }^{18}$ Saari M, Vuorre I, Nieminen H: Fuchs's heterochromic cyclitis: a simultaneous bilateral fluorescein angiographic study of the iris. Br J Ophthalmol 1978, 62: 715-21.

${ }^{19}$ Sugar HS: Heterochromia iridis with special consideration of its relation to cyclitic disease. Am J Ophthalmol 1965, 60: 1-18.
${ }^{20}$ Chandler PA and Grant WM: Lectures on Glaucoma. Lea \& Febinger, Philadelphia 1965.

${ }^{21}$ Loewenfeld IE and Thompson S: Fuchs's heterochromic cyclitis. A critical review of the literature I. Clinical characteristics of the syndrome. Surv Ophthalmol 1973, 17: 394-457.

${ }^{22}$ Henkind P: Angle vessels in normal eyes. A gonioscopic evaluation and anatomic correlation. $\mathrm{Br} \mathrm{J}$ Ophthalmol 1964, 48: 551-7.

${ }^{23}$ Kimura SJ, Hogan MJ, Thygeson P: Fuchs' syndrome of heterochromic cyclitis. Arch Ophthalmol 1955, 54: 179-86.

${ }^{24}$ Ward DM and Hart CT: Complicated cataract extraction in Fuchs's heterochromic uveitis. $\mathrm{Br} \mathrm{J}$ Ophthalmol 1967, 51: 530-8.

${ }^{25}$ Mills KB and Rosen ES: Intraocular lens implantation following cataract extraction in Fuchs' heterochromic uveitis. Ophthalmic Surg 1982, 13: 467-9.

${ }^{26}$ Gee SS and Tabbara KF: Extracapsular cataract extraction in Fuchs' heterochromic iridocyclitis. Am J Ophthalmol 1989, 108: 310-14.

${ }^{27}$ Jakeman CM, Jordan K, Keast-Butler J, Perry S: Cataract surgery with intraocular lens implantation in Fuchs' heterochromic cyclitis. Eye 1990 , 4: 543-7.

Hooper PL, Rao NA, Smith RE: Cataract extraction in uveitis patients. Surv Ophthalmol 1990, 35: $120-44$

${ }^{29}$ Franceschetti A: Heterochromic cyclitis (Fuchs' Syndrome) Am J Ophthalmol 1955, 39: 50-8. 